\title{
A new upper divertor with internal coils for ASDEX Upgrade - status of the project
}

\author{
A. Herrmann, M. Teschke, I. Zammuto, M. Cuevas, M. Dibon, A. Kallenbach, T. Lunt, V. Rohde, G. Schall, \\ T. Vierle, S. Vorbrugg, M. Weißgerber, H. Zohm and the ASDEX Upgrade team
}

\section{Max-Planck-Institut für Plasmaphysik, D-85748 Garching, Germany}

ASDEX Upgrade is a tokamak that can be operated with the strike lines in the upper and/or the lower divertor. In 2016 a project was started to develop and install a new upper divertor with internal coils and an in-vessel cryo-pump. The aim is to investigate alternative magnetic configurations that may facilitate the access to detachment via an enhanced poloidal flux tube expansion and/or connection length. To realize the envisaged magnetic configurations two internal coils operated with up to $52 \mathrm{kAt}$ will be installed near the upper outer strike line. Since the conceptual design was presented critical aspects of the project were identified and investigated in detail. These are the vessel integration, the coil concept, the forces, in particular during disruptions, an intrinsic safe power supply, the cryo-pump and last but not least, operation with co and counter magnetic field. The present status of each of the components is presented in the paper.

Keywords: ASDEX Upgrade, Divertor, magnetic configurations, alternative concepts, electromagnetic forces

\section{Introduction}

ASDEX Upgrade (AUG) is a tokamak that can be operated with the strike lines in the upper and/or the lower divertor. The physics program was and is concentrated on magnetic configurations with the strike lines in the lower divertor. The lower divertor evolved from an open divertor towards closed divertor configuration with vertical targets as it is documented in [1]. The realization of alternative magnetic configurations able to spread the parallel heat flux reaching the divertor region over a larger area requires in-vessel coils near to the strike line region $[2,3]$.

In 2016 a project was started to develop and install a new upper divertor with internal coils and an invessel cryo-pump coming into operation in 2022. The conceptual design was presented in [4]. The requirements from the conceptual design are updated in the following list:

1. Two divertor coils with up to $52 \mathrm{kAt}$ each, allowing to access alternative divertor configurations [2]. $52 \mathrm{kAt}$ are realized in the preliminary design by a 4 turn coil fed with $13 \mathrm{kA}$.

2. The coils are operated in both polarities, but with currents in opposite directions. To fulfill the physics requirements an imbalanced feeding of the coils with up to $\Delta \mathrm{I}_{\text {coil }}<10 \mathrm{kAt}$ is required [2].

3. The physics program for the lower divertor should not be restricted. Existing reference discharges in LSN configuration have to be compatible with the new structure of the upper divertor.

4. Pumping speed of at least $S_{\text {eff }}=15 \mathrm{~m}^{3} / \mathrm{s}$ in the private flux region to ensure a pumping capability comparable to the existing cryo-pump in the lower divertor.

5. Hardening of the upper divertor target structure to cope with the high heating power of AUG with tolerable leading edge effects for both helicities.

During the preliminary design phase critical aspects of the project were identified and investigated in detail. These are the in vessel integration, the coil concept, the

60 forces, in particular during disruptions, an intrinsic safe 61 power supply, the cryo-pump and last but not least, 62 operation with co and counter magnetic field.

63 The next section presents general aspects of in64 vessel integration before the forces, particular induced 65 during disruptions, and the consequences for the coil 66 design are discussed in section 3. Section 4 presents 67 coil options under investigation. The status of the cryo68 pump is discussed in section 5. Finally, the target 69 concept for bidirectional operation is presented in 70 section 6 before a summary is given.

\section{2. In-vessel integration aspects}

The design of the components evolved during the preliminary design phase. The present status is shown in Fig. 1.

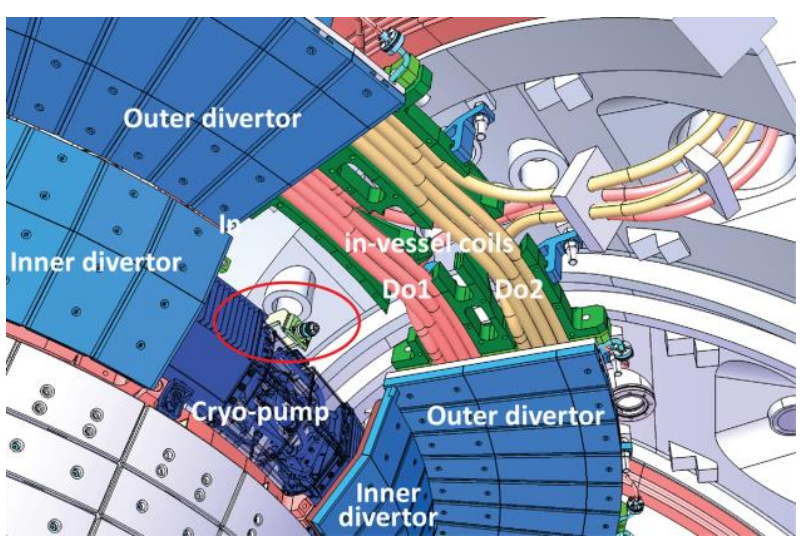

Fig. 1 CAD view into the upper divertor with two in-vessel coils, Do1 and Do2, close to the outer strike line, a cryopump behind the inner divertor and the arrangement of tungsten coated target tiles with minimized gap size. Regions with tight geometrical constraints are circled in red.

The preliminary design was checked for geometrical configuration compatibility with other invessel components of AUG. The as-is state was documented with a 3D scan of the inner vessel during one of the recent openings when the present divertor 
111 Fig. 2 Scheme of the power supply with one power supply for

components were removed. It revealed that there are component envelops overlap in regions around the inner ports and behind the upper outer divertor as marked in Fig. 1, mainly due to components that are not included in the 3D-CAD documentation, such as cables and a few in-vessel flanges. As a consequence the upper outer divertor is moved down by additional $15 \mathrm{~mm}$ and the cryo-pump is slightly modified without reducing the pumping speed.

\section{Forces due to in-vessel coils and power supply}

The two in-vessel coils have to be operated with up to $52 \mathrm{kAt}$ to realize the required magnetic configurations. If both coils are operated with a parallel current feeding the resulting force onto the coil support structure and the vessel would be in the order of the vessel weight, i.e unacceptable high [4]. Operation with tolerable forces requires an anti-parallel coil feeding with a maximum imbalance between the two coils of $\Delta \mathrm{I}_{\text {coil }}<10 \mathrm{kAt}$, also in case of a failure of the power supply. This is ensured by a hardwired antiparallel feeding of the two coils combined with an imbalanced current fed by a separate power supply as shown in Fig. 2, and discussed in detail in [5].

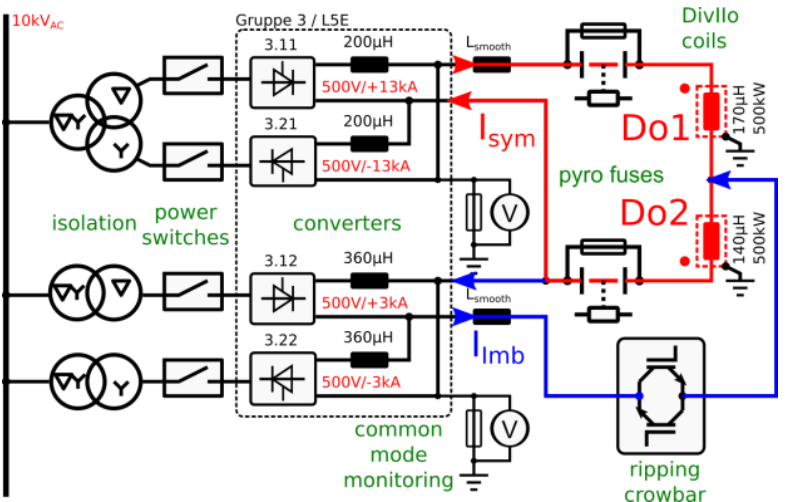
the asymmetric feeding of both coils (red) and an additional feeding of a single coil with up to $3 \mathrm{kA}$ to realize the imbalance required for the magnetic configurations (blue).

A main focus during the preliminary design phase was on disruption issues. Disruptions are unavoidable in a present day tokamak and are part of the physics program. They result in a strong change of magnetic fields due to the decay and the movement of the plasma current. Both effects induce voltages inside in-vessel components. If there is a closed loop, currents are driven and interact with the constant toroidal field. To estimate the $\mathrm{dB} / \mathrm{dt}$ to be applied to the force calculation magnetic data from discharges of the last AUG campaigns were analysed and a value of $\mathrm{dB} / \mathrm{dt}=80 \mathrm{~T} / \mathrm{s}$ was found to be the worst case in the upper part of the vessel [5]. Such a high $\mathrm{dB} / \mathrm{dt}$ acting on the Div-IIo results in $\mathrm{U}_{\text {ind }} \approx 1 \mathrm{kV} /$ turn [5]. This induced voltage might result in a problem for a coil in particular with a larger number of turns if the electric strength is too low.

To define the forces acting on the coils and the support structure during disruptions as well as due to failures inside the coil and the power supply a 2D electromagnetic model was set up. It uses measured magnetic data and external coil currents as input data and calculates induced voltages, the poloidal field distribution, currents and forces [5]. This model was carefully cross-checked against AUG disruptions and afterwards applied to calculate the consequences of failures due to disruptions. Two critical failures are identified. (i) The induced voltage causes an arc, i.e. a short-circuit between two turns. The resulting current through the single turn closed loop results in forces of about $F_{z}=225 \mathrm{kN}$. (ii) The power supply for the imbalance feeding suffers an external short circuit during disruptions and a high current flows through the coil with 4 turns resulting in a force of $F_{z}=308 \mathrm{kN}$. Both failures have to be avoided by design - (i) by installing a conductor with an electrical screen; (ii) by developing an 'intelligent fuse' opening the connection between power supply and coil in case of increasing currents. The 2D electromagnetic model is also used to estimate the influence of the new upper divertor on the plasma break down behaviour.

The maximum force acting onto a single coil is $\mathrm{F}_{\mathrm{z}}=105 \mathrm{kN}$ during normal operation with $52 \mathrm{kAt}$ and $\mathrm{F}_{\mathrm{z}_{-} \text {dis }}=37 \mathrm{kN}$ remaining force during a disruption.

The forces gained by the 2D model are transferred into a 3D mechanical FEM model to calculate stresses and displacements of divertor components including the coil support structure for normal operation and disruptions. If resulting stresses and displacements are too high, the design of components is modified to mitigate the overload.

In addition, a 3D electromagnetic FEM model is used to deduce the forces on components that are not toroidally symmetric, such as modules of the cryopump.

\section{Coil options}

Since the coil design and the forces are interconnected the design was improved in an iterative approach considering the following constraints and criteria:

- Electrical strength and disruption induced voltage.

- An existing power supply with up to $27 \mathrm{kA} / 500 \mathrm{~V}$ should be used.

- The maximum temperature increase of the conductor should be kept below $\Delta \mathrm{T}=60 \mathrm{~K}$ for $5 \mathrm{~s}$ of operation.

- The fill-factor of the conductor, i.e. the ratio of the usable copper cross section and the cross section of the conductor.

- Handling and in-vessel installation.

From force, geometric and power supply considerations two coils with 4 turns each arranged in a single layer (see Fig. 1) fed with $13 \mathrm{kA}$ are found as optimum.

Isolation tests performed in vacuum with a pure copper conductor embedded in an isolating casing made from PEEK reveals that discharges between adjacent isolated conductors cannot be avoided at voltages above about $150 \mathrm{~V}$. These discharges might trigger a high current arc, causing a permanent shortcircuit that restricts operation of the coil and the 
experiment due to the high disruption forces as discussed in section 3. Consequently, a coil design should have an electrical screen between single turns to avoid the inter-turn short circuit. In addition, the electrical screen allows to detect a degradation of the insulation around the conductor [5]. Two types of water cooled conductors were considered in more detail. A quadratic copper conductor that has to be embedded in an insulating and electrical screening casing and a Tefzel insulated circular copper conductor embedded in a stainless steel casing (TIC). The invessel handling of quadratic copper conductor is simpler than the co-axial TIC conductor but needs an additional electrical screen that is already part of the design of the TIC conductor. The TIC conductor is inherent safe against winding short circuit and therefore it is the preferred solution. It is also compatible with the maximum force, $F_{z}=(105+36) \mathrm{kN}$ or $141 \mathrm{kN} / 40 \mathrm{~m} / 0.01 \mathrm{~m}=0.35 \mathrm{MPa}$, that has to be transferred from the copper conductor via the Tefzel insulation, tensile strength $\sigma_{\mathrm{t}} \approx 17 \mathrm{MPa}$, onto the protecting tube.

To qualify the electrical and mechanical properties as well as the installation procedure of the TIC conductor prototypes were ordered from an external company. They are under examination at IPP. Electrical and thermo-mechanical tests were started. The electrical strength was successfully tested up to $10 \mathrm{kV}$. A method was developed for non-destructive detection of small voids by applying an AC high voltage and measuring the transferred charge. This helps to detect modifications of the insulation due to thermo-mechanical and bending tests.
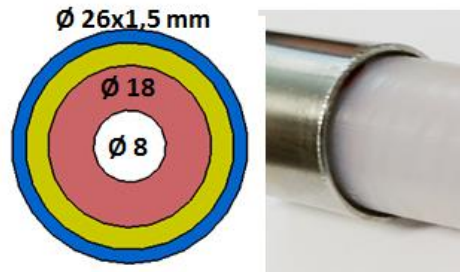

Fig. 3 Tefzel isolated conductor in a stainless steel protection tube.

For the coil layout and the electrical feed through the thermal expansion of the conductor is a significant parameter. During the $5 \mathrm{~s}$ load phase, the copper conductor warms up by $\Delta \mathrm{T}=60 \mathrm{~K}$ and the stainless steel jacket stays cool. The first thermo-mechanical tests reveal that the thermal expansion of the copper is transferred to the stainless steel protection tube so that the difference in the length expansion between the copper and the stainless steel tube is only about $\Delta \mathrm{x}=300 \mu \mathrm{m}$. This is explained by the radial pressure imposed by the manufacturing process being further enhanced during the load phase due to the thermal expansion of the copper. This behaviour is qualitatively understood by an analytical model and will be analysed by a more sophisticated FEM model considering the strong nonlinear behaviour of Tefzel. At present, the tests are repeated with a virgin and longer TIC conductor to verify the results and to validate the model that predicts that the difference in the expansion is independent from the total length of the conductor.

An important criterion for the choice of a solution is the handling and installation of the coil. The design of AUG has not foreseen that the vacuum vessel can be separated into two parts for installation of large components, such as coils. Access to the vessel is via a $40 \times 80 \mathrm{~cm}^{2}$ manhole. This port is large enough to bring components extending over a sector into the vessel. It does not allow bringing a complete coil into the vessel and the in-vessel coils have to be wound inside the vessel. At present 3 options are under investigation:

1. An about $60 \mathrm{~m}$ long conductor is placed outside the vessel. It will be inserted into the bending assembly placed inside the vessel. The whole coil including the current feeding is bent inside the vessel. This is most challenging due to the small bending radii near to the current feeding of about $200 \mathrm{~mm}$.

2. Only the toroidal part of the coil is bent inside the vessel. This can be done as a four turn winding or by bending four separate turns. For this solution joints are needed to connect the pieces of the coil.

3. The coil pieces are bent outside the vessel and will be connected inside the vessel with joints. Here the longest possible component that can be fed through the port is $3 \mathrm{~m}$. This solution needs at least 3 joints per turn.

The favorable option is option 1 because it needs no joints inside the vessel. Option 2 needs at least 2 joints/coil but is less challenging with respect to the bending of the conductor with about $1500 \mathrm{~mm}$ bending radius. Option 3 avoids the bending inside the vessel but needs a large number of joints. In addition to the uncertain reliability of joints this option needs more space for the coil because of the larger diameter of the joints compared to the conductor itself.

The bending as well as the joint technology is under investigation. Bending tests are performed in collaboration with an external company. The joint qualification is done in-house. The tools for high frequency- soldering and orbital welding are identified and tested together with external companies. Results enabling a final decision on the option to be realized are expected until summer 2019.

\section{Cryo-pump}

Density control in the divertor region of the new upper divertor should be realized by a cryo-pump behind the upper divertor with $50 \mathrm{~m}^{3} / \mathrm{s}$ pumping speed, see Fig. 1. The $30 \mathrm{~mm}$ wide slit between the inner and outer divertor ensures an effective pumping speed for hydrogen isotopes of about $20 \mathrm{~m}^{3} / \mathrm{s}$ in the upper divertor region.

The design of the cryo-pump is based on the positive experience with the cryo-pump in the lower outer divertor of AUG [6]. It consists of 7 modules arranged toroidally around the torus. The cryo-pump is located in front of the inner most ports and blocks a direct view to the plasma. For dedicated diagnostics the cryo-pump design opens this direct view. That is why 2 types of modules with different toroidal extension will be installed. 


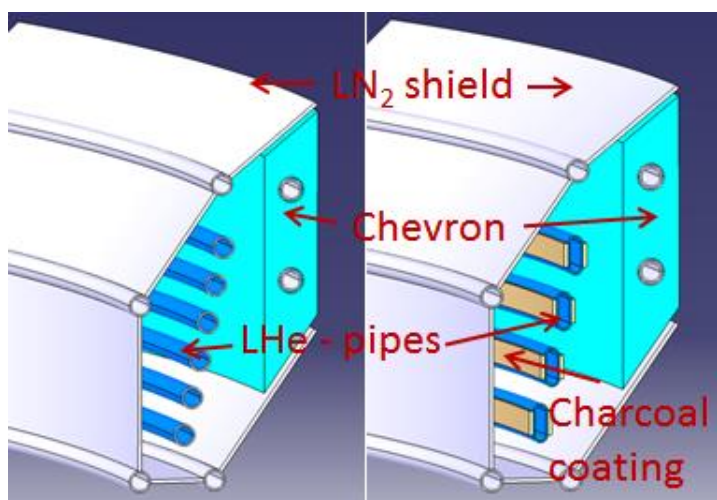

Fig. 4 Cryo-pump layouts left the standard, right with the option to coat the He pipes with activated charcoal for $\mathrm{He}$ pumping.

An adjustment of the pumping speed will be realized by a 3 pass valve in the He-cooling pipes at about $2 / 3$ of the toroidal circumference allowing a disconnection of the last $1 / 3$ of the cryo-pump. A cross section of the cryo-pump is shown in Fig. 4. At present the possibility to coat the He-pipes with activated charcoal for He pumping is under investigation. The coating technology developed for ITER [7] requires flat areas and cannot be applied to round tubes. Consequently, the cryo-pump design for He pumping has a reduced number of He pipes, see Fig. 4. This allows using larger tubes $(16 \times 1$ instead of $12 \times 1)$ that are formed to a rectangle cross section (20x8) enabling the coating. This design would allow an effective pumping speed for $\mathrm{He}$ of about $10 \mathrm{~m}^{3} / \mathrm{s}$.

The cryo-supply outside the vessel is designed. A common cryo-supply for $\mathrm{LHe} / \mathrm{LN}_{2}$ is used for the upper and lower cryo-pump. Near to the torus a connection box will be installed to separate the cryosupply for the upper from the lower cryo-pump.

\section{Target design and shaping}

AUG is a tokamak experiment with a weak cooling of components in plasma contact. The targets are clamped onto stainless steel water cooled support structures. The target thickness gives a limit for the heat absorbing capacity. It is between $15 \mathrm{~mm}$ for tungsten targets and about $30 \mathrm{~mm}$ for graphite. The tungsten coated graphite targets for the upper divertor are designed accordingly. However, there are two peculiarities for the design of the upper divertor targets. (i) There is no space to place fixing elements behind the cooling structure. (ii) The divertor cooling structure for the outer targets is part of the well aligned toroidally closed stiff coil support structure. There is no need to compensate a misalignment between divertor modules by target tilting. This offers the possibility to operate in the upper divertor with magnetic configurations in both directions of helicity. Anyhow, also if there is no need for target tilting, an ideal divertor with zero gap size cannot be realized due to manufacturing tolerances and the need for small toroidal and poloidal gaps to allow thermal expansion. The effect of a non-ideal divertor with $\mathrm{d}=1 \mathrm{~mm}$ gaps in between targets and $\mathrm{u}= \pm 0.1 \mathrm{~mm}$ alignment tolerance was taken as design criteria to define an
360 optimal target chamfering minimizing leading edge 361 effects.
362

363 364
Fig. 5. Geometry and input data used for optimization of the target chamfering.

The geometry is shown in Fig. 5. Here $\alpha_{m}$ is the maximum tolerable pitch angle. The real pitch angle $\alpha<\alpha_{\mathrm{m}}$ depends on the magnetic configuration and varies between 0.12 and $3.7 \mathrm{deg}$ for the envisaged magnetic configurations at the strike line. $\mathrm{h}$ and $\mathrm{s}$ are free parameters for optimization. A number of 2D FEM thermal calculations were carried out to parametrize the peak heat load for a set of $\alpha$ values varying $h$ and $\mathrm{s}$ with fixed $\mathrm{d}, \mathrm{u}$, and $\alpha_{\mathrm{m}}$. A moderate chamfering with $\mathrm{s}=7 \mathrm{~mm}$ and $\mathrm{h}=1 \mathrm{~mm}$ was found to be the optimum. In comparison to this a roof-like shape with $s=w / 2$, where $\mathrm{w}$ is the width of the tile, was also considered. Such a target might be uniformly loaded for $\alpha=\alpha_{m}$ but it is strongly overheated for $\alpha<<\alpha_{\mathrm{m}}$.

A new target clamping solution was designed, that allows fixing the target from the plasma side as shown in Fig. 6. The basic concept is that the clamping mechanism including the disc springs is preinstalled in the divertor cooling structure. The target is fixed with a pretension defined by the disc springs. Two types of target fixings are foreseen. In the region with a high heat load the target is fixed by placing it and turning the eccentric tappets inside the sliding blocks. The neighbor tiles placed left and right from the central highly loaded tile need access from the front side for fixing with screws. Both target fixings are manufactured and tested as prototypes.

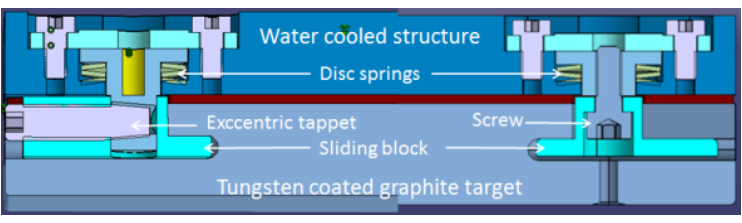

Fig. 6. 3D cut showing the clamping mechanism for the target tiles. Left, fixing applied in the high heat load region, right, with front side access applied in the low heat load region. The target thickness is $20 \mathrm{~mm}$.

\section{Summary and outlook}

During the preliminary design phase the components of the Div-IIo project were detailed, checked and adjusted for geometrical compatibility with the existing components. Disruptions were identified as causing the highest forces. Avoiding an overloading of components requires an intrinsic safe design of the power supply and the conductor. The bending and joint concept will be defined on the basis of further test at the end of 2018.

Acknowledgements: This work has been carried out within the framework of the EUROfusion Consortium and has received funding from the Euratom research 
411 and training programme 2014-2018 under grant

412 agreement No 633053 in the framework of the MST 2

413 programme. The views and opinions expressed herein

414 do not necessarily reflect those of the European

415 Commission.

\section{References}

417 [1] A. Herrmann, et al., Fusion Science and 418 Technology, 44 (2003) 569-577.

419 [2] T. Lunt, et al., Nuclear Materials and Energy, 12 420 (2017) 1037-1042.

421 [3] T. Lunt, et al., Nuclear Materials and Energy, PSI 4222018 proceedings (2019).

423 [4] A. Herrmann, et al., Fusion Eng. Des., 123 (2017) 424 508-512.

425 [5] M. Teschke, et al., Fusion Energy and Design, Soft 426 conference 2018 (2018).

427 [6] B. Streibl, et al., Fusion Technology (Proc. of the 428 19th Symposium on Fusion Technology, Lisbon, 429 1997), 1997, pp. 427-430.

430 [7] C. Day, Colloids and Surfaces A: Physicochemical 431 and Engineering Aspects, 187-188 (2001) 187-206. 